taken at deliveries (91\%) and the results stored (100\%). However, there are some areas where improvements are needed such as the documentation of consent, reasons for FBS, results, and the documentation of review at 30 minutes post FBS.

Conclusion Despite being indicated in the trust guideline, some of the essential documentations regarding FBS are still missing. Therefore, for these cases any future claims for cerebral palsy would be indefensible. FBS proforma was developed and are used for all FBS cases. This recommendation was subsequently adopted by $\mathrm{DMH}$.

\section{PL.100 TIME TO DISCHARGE FOLLOWING UNCOMPLICATED ELECTIVE OR EMERGENCY CAESAREAN SECTION: IS PATIENT MOTIVATION THE MAJOR DETERMINING FACTOR?}

doi:10.1136/archdischild-2013-303966.282

S Rundle, J Ash, T Kay. Royal Devon and Exeter Foundation NHS Trust, Exeter, UK

Anticipated increase in the rate of Caesarean birth following the publication of recent National Institute of Health and Clinical Excellence guidance ${ }^{1}$ prompted investigation into the causes of delay in discharge from hospital following an uncomplicated elective or emergency Caesarean section. A retrospective audit of the patient health record at the Royal Devon and Exeter Hospital revealed that time to discharge appeared to be independent of parity, urgency of delivery (elective or emergency Caesarean section) or intended feeding method (breast of artificial feeding).

In patients without medical or surgical complications following Caesarean delivery, it was expected that delay in medical and midwifery staff performing routine care needs would be responsible for any delay in discharge. Routine and measurable care needs, identified as being compulsory prior to discharge were: 1 . Removal of the indwelling urinary catheter and successful trial without catheter (TWOC), 2. Documented confidence and competence at breastfeeding, for those choosing this method, 3. Ability to administer low molecular weight thromboprophylaxis independently of a midwife or health professional, and, 4. Ability to self-administer postoperative analgesia. On no identifiable occasion did unnecessary delay in the performance of these needs adversely affect time to discharge.

These results suggest that in our unit patient motivation factors as well as individual expectation of the recovery period may be the biggest determinants of time to discharge following Caesarean section.

\section{REFERENCE}

1. National Institute for Health and Clinical Excellence. NICE clinical guideline 132. Caesarean Section: November 2011

\section{Pregnancy Outcome Posters}

\section{PP.01 PERINATAL OUTCOME OF IUGR PREGNANCIES WITH NORMAL AND ABNORMAL DOPPLER STUDIES - THE PROSPECTIVE MULTICENTRE PORTO TRIAL}

doi:10.1136/archdischild-2013-303966.283

J Unterscheider, S Daly, MP Geary, MM Kennelly, FM McAuliffe, K O'Donoghue, A Hunter, JJ Morrison, G Burke, P Dicker, EC Tully, FD Malone. Perinatal Ireland Research Consortium, Dublin, Ireland

Objective The objective of this analysis, as part of the multicentre prospective PORTO Trial, was to describe and compare perinatal outcomes of IUGR pregnancies with normal and abnormal umbilical artery (UA) Doppler studies.

Study design The PORTO Trial recruited over 1,100 consecutive ultrasound-dated singleton IUGR pregnancies, defined as EFW $<10^{\text {th }}$ centile. All fetuses underwent serial sonographic assessment until birth. Perinatal outcomes were documented for all participants.
Abstract PP.01 Table 1 Comparison of groups with normal and abnormal UA Doppler

\begin{tabular}{llll}
\hline & Normal Doppler & Abnormal Doppler & p-value \\
\hline GA (enrolment) & $30.4 \pm 4.0$ & $29.6 \pm 3.8$ & 0.0007 \\
GA (delivery) & $38.4 \pm 2.2$ & $36.6 \pm 3.5$ & $<0.0001$ \\
Birthweight & $2641 \pm 553$ & $2203 \pm 725$ & $<0.0001$ \\
NICU admission & $121(20 \%)$ & $191(37 \%)$ & $<0.0001$ \\
Adverse perinatal outcome* & $11(2 \%)$ & $49(10 \%)$ & $<0.0001$ \\
Perinatal mortality & $4(0.7 \%)$ & $6(1 \%)$ & 0.3652 \\
Induction & $303(50 \%)$ & $217(42 \%)$ & 0.0110 \\
CS & $190(32 \%)$ & $256(50 \%)$ & $<0.0001$ \\
Instrumental & $66(11 \%)$ & $41(8 \%)$ & 0.1025 \\
NVD & $350(58 \%)$ & $215(42 \%)$ & $<0.0001$ \\
\hline
\end{tabular}

${ }^{*}$ composite outcome of IVH, PVL, HIE, NEC, BPD, sepsis and death

Results Of the 1,118 recruited patients, 606 (54\%) had normal UA Doppler studies and 512 (46\%) had abnormal UA Dopplers, defined as UA PI $>95^{\text {th }}$ centile or AREDF. The group with abnormal UA Doppler was delivered earlier and more commonly by CS, had more admissions to NICU and adverse perinatal outcomes (Table 1). The 4 mortalities with normal Doppler all had EFW $<3^{\text {rd }}$ centile.

Conclusion IUGR fetuses with normal UA Doppler studies have better perinatal outcomes than those with abnormal UA Doppler, however adverse perinatal outcome can occur with normal UA in more severe IUGR cases (EFW $<3^{\text {rd }}$ centile).

\section{PP.02 EFFECT OF METHOD AND GESTATIONAL AGE AT TERMINATION OF PREGNANCY ON FUTURE OBSTETRIC AND PERINATAL OUTCOMES: A RETROSPECTIVE COHORT STUDY}

doi:10.1136/archdischild-2013-303966.284

${ }^{1,2}$ A Woolner, ${ }^{1,2}$ S Bhattacharya, 'S Bhattacharya. 'University of Aberdeen, Aberdeen, UK; ${ }^{2}$ NHS Grampian, Aberdeen, UK

Induced abortion (IA) is believed to increase the risk of spontaneous preterm labour. Few studies have investigated the impact of method used (medical versus surgical) or the gestational age at IA. In a population based retrospective cohort study using data from the Aberdeen Maternity Neonatal Databank, the outcome of a subsequent viable pregnancy in 3186 women who underwent IA in their first pregnancy was compared with 42446 primigravid women. The exposed cohort was stratified according to method and gestational age at IA. Perinatal outcomes following medical IA was compared to those following surgical IA, as well as those in primigravid women. Similarly, women who underwent IA at $<13$ weeks were compared to women with history of IA at $>=13$ weeks and primigravid women. Univariate and multivariate logistic regression adjusted for maternal age at delivery, smoking and socioeconomic status were used to analyse the data. No statistically significant association was found between previous IA and spontaneous preterm labour ( $\mathrm{aOR} 1.05(0.88-1.27))$. Neither the method of termination ( $\mathrm{aOR} 0.95$ (0.72 to 1.25)) nor gestational age (aOR 1.00 (0.99 to 1.00)) at IA appeared to affect the risk of spontaneous preterm delivery. IA increased the risk of antepartum haemorrhage ( $p<0.001$; aOR 1.22 (1.09 to 1.36) in the next pregnancy. Previous IA appeared to protect against pregnancy induced hypertension (aOR $0.67(0.60-0.74)$ ). Method and gestational age at IA largely did not affect future obstetric and perinatal outcomes. Evidence remains conflicting on pregnancy outcomes following termination of pregnancy. 